PROCEEDINGS OF THE

AMERICAN MATHEMATICAL SOCIETY

Volume 134, Number 7 , Pages 2027-2035

S 0002-9939(05)08209-2

Article electronically published on December 19, 2005

\title{
HYPER-REFLEXIVITY OF FREE SEMIGROUPOID ALGEBRAS
}

\author{
FRÉDÉRIC JAËCK AND STEPHEN C. POWER \\ (Communicated by David R. Larson)
}

\begin{abstract}
As a generalization of the free semigroup algebras considered by Davidson and Pitts, and others, the second author and D.W. Kribs initiated a study of reflexive algebras associated with directed graphs. A free semigroupoid algebra $\mathcal{L}_{G}$ is generated by a family of partial isometries, and initial projections, which act on a generalized Fock space spawned by the directed graph $G$. We show that if the graph is finite, then $\mathcal{L}_{G}$ is hyper-reflexive.
\end{abstract}

\section{INTRODUCTION}

The free semigroup algebra $\mathcal{L}_{n}$ is the weakly closed operator algebra generated by the left regular Hilbert space representation of the free semigroup on $n$ generators. These algebras, which are free analogues of the operator algebra $H^{\infty}$, have been considered in detail by Davidson, Pitts, Popescu and others ([7, [8, [9, 1], 12, [13). As a generalisation of this class the second author and D.W. Kribs in [10], [11] have considered reflexive algebras associated with directed graphs, the so-called free semigroupoid algebras. Such an algebra $\mathcal{L}_{G}$ is generated by a family of partial isometries and initial projections which act on a generalized Fock space $\mathcal{H}_{G}$ spawned by a directed graph $G$.

Let $\mathcal{B}(\mathcal{H})$ be the algebra of all bounded operators on the separable Hilbert space $\mathcal{H}$, and let $\mathcal{A}$ be a WOT-closed subspace of $\mathcal{B}(\mathcal{H})$. For a given operator $T \in \mathcal{B}(\mathcal{H})$, consider the following two quantities:

$$
d(T, \mathcal{A}):=\inf _{A \in \mathcal{A}}\|T-A\| \text { and } \beta_{\mathcal{A}}(T):=\sup _{(Q, P) \in \mathcal{F}_{\mathcal{A}}}\|Q T P\|,
$$

where $\mathcal{F}_{\mathcal{A}}$ is the set of all pairs of projections annihilating $\mathcal{A}$, that is,

$$
\mathcal{F}_{\mathcal{A}}=\{(Q, P) ; Q A P=0, \text { for all } A \in \mathcal{A}\} .
$$

Recall that $\mathcal{A}$ is said to be a reflexive space if $\beta_{\mathcal{A}}(A)=0$ entails $A \in \mathcal{A}$. In this case $\beta_{\mathcal{A}}(T)$ can also be viewed as a distance from $T$ to $\mathcal{A}$. It is easy to check that $\beta_{\mathcal{A}}(T) \leq d(T, \mathcal{A})$ while if the two distances are equivalent, then the space $\mathcal{A}$ is said to be hyper-reflexive. The smallest constant $C>0$ such that $d(T, \mathcal{A}) \leq C \beta_{\mathcal{A}}(T)$ for every operator $T$ is known as the hyper-reflexivity constant for $\mathcal{A}$. This metric

Received by the editors February 10, 2005.

2000 Mathematics Subject Classification. Primary 47L75.

Key words and phrases. Free semigroupoid, reflexive algebra, directed graph, hyper-reflexive.

This work is part of the research program of the network "Analysis and Operators" supported by the European Community's Potential Program under HPRN-CT-2000-00116 (Analysis and operators).

(C)2005 American Mathematical Society Reverts to public domain 28 years from publication 
strengthening of reflexivity was examined by Arveson in [2]. See Davidson [5] for additional discussions.

There are few systematic tools available to prove hyper-reflexivity and as a result the variety of known hyper-reflexive algebras is limited. The hyper-reflexivity of $\mathcal{L}_{1}$, which is the algebra $H^{\infty}(\mathbb{T})$, realized as the Toeplitz algebra on $H^{2}(\mathbb{T})$, was established by K.R. Davidson [6]. By different methods Davidson and Pitts proved that the remaining free semigroup algebras $\mathcal{L}_{n}$ are hyper-reflexive. (Reflexivity was shown earlier by Arias and Popescu [1] and an alternative short proof can be found in Kribs and Power [10.) Bercovici [3] subsequently obtained a new approach to hyper-reflexivity by means of a very general result for spaces with property $X_{\theta, \gamma}$. This property holds for operator algebras whose commutants contain a pair of commuting isometries with orthogonal ranges and so is immediately applicable to free semigroupoid algebras as well as free semigroup algebras. In the present paper we deal with the case of finite graphs whose algebras do not have this property and combining the approaches we obtain the following main result.

Theorem. The free semigroupoid algebra $\mathcal{L}_{G}$ of a finite directed graph $G$ is hyperreflexive.

In fact our proof also shows that $\mathcal{L}_{G} \otimes \mathcal{B}(\mathcal{K})$ is hyper-reflexive and we remark on such complete hyper-reflexivity in Section 3.

Free semigroupoid algebras may be defined as follows. Let $G$ be a directed graph with vertex set $\mathcal{V}(G)$ and edge set $\mathcal{E}(G)$, each set being finite or countable. A directed path $p$ in $G$ is either a single vertex (considered as a degenerate path) or an $N$-tuple of edges $p=e_{N} e_{N-1} \ldots e_{1}$ such that the source vertex of $e_{i+1}$ is equal to the range vertex of $e_{i}$ for each $i$. The length $|p|$ of the path $p$ is the number of edges in $p$. The discrete semigroupoid $\mathbb{F}_{+}(G)$ of $G$ is the set of all finite directed paths with the partially defined associative product arising from concatenation. Note that $\mathbb{F}_{+}(G)$ is a multiplicatively closed subset of the (free) path groupoid $\mathbb{F}(G)$ of $G$ which is obtained in a similar way from the set of vertices and all finite paths (or words) in the edges $e$ and their formal inverses $e^{-1}$. These words are free except for the groupoid relations $e^{-1} e=x, e e^{-1}=y$, where $e$ has $x, y$ as source and range vertices. Also $\mathbb{F}_{+}(G)$ is a unital subset in that it contains the units (vertices) of the groupoid $\mathbb{F}(G)$. These observations and the parallel with free semigroups support the use here of the term free semigroupoid.

The path semigroupoid $\mathbb{F}_{+}(G)$ gives rise in a natural way to a family of partial isometries: Let $\mathcal{H}$ be the Hilbert space with basis $\left\{\xi_{w}: w \in \mathbb{F}_{+}(G)\right\}$ and define the partial isometry $L_{w}$ for which

$$
L_{w} \xi_{w^{\prime}}= \begin{cases}\xi_{w w^{\prime}} & \text { if } w w^{\prime} \text { is defined } \\ 0 & \text { otherwise }\end{cases}
$$

If $x \in \mathcal{V}(G)$, then $L_{x}$ is the projection onto the closed space spanned by $\left\{\xi_{w}: w=\right.$ $x u\}$. Thus, $L_{x}$ is the initial projection $L_{e}^{*} L_{e}$ for any $e$ with $e=e x$.

Definition 1.1. Let $G$ be a countable directed graph. Then the free semigroupoid algebra $\mathcal{L}_{G}$ is the WOT-closed algebra generated by $\left\{L_{e}: e \in \mathcal{E}(G) \cup \mathcal{V}(G)\right\}$.

In particular, a graph with a single vertex and $n$ edges gives rise to the free semigroup algebra $\mathcal{L}_{n}$.

We would like to thank Ken Davidson for some helpful comments on a preliminary version of this paper. 


\section{Some GENERAL RESUlts ABOUt HYPER-REFLEXIVITY}

In this section, we recall some key results about hyper-reflexivity. Moreover, we derive some auxiliary results that we need for our proof, including the fact that certain partial inflations of a hyper-reflexive algebra are again hyper-reflexive.

Theorem 2.1 (Davidson [6]). The algebra $H^{\infty}(\mathbb{T})$ realized as the algebra of Toeplitz operators acting on $H^{2}(\mathbb{T})$ is hyper-reflexive, with constant at most 19.

Recall that the WOT-continuous functionals on $\mathcal{B}(\mathcal{H})$ are exactly the functionals of the type $\phi=\sum_{i=1}^{n}\left[x_{i} \otimes y_{i}\right]$ where the elementary functionals $[x \otimes y]$ are defined by their action on an operator $T$ by $[x \otimes y](T)=(T x, y)$. A WOT-closed space $\mathcal{M}$ is said to have property $\left(\mathbb{A}_{1}\right)$ if every WOT-continuous functional $\phi$ on $\mathcal{M}$ can be written $\phi=[x \otimes y]$ for some $x, y \in \mathcal{H}$. Moreover, given $r>0, \mathcal{M}$ is said to have property $\left(\mathbb{A}_{1}(r)\right)$ if for any $\epsilon>0$, one can find vectors $x, y \in \mathcal{H}$ such that $\phi=[x \otimes y]$ and $\|x\|,\|y\| \leq(r+\epsilon)\|\phi\|^{1 / 2}$.

Theorem 2.2 (Davidson [6]). Suppose $\mathcal{A}$ is a hyper-reflexive subspace of $\mathcal{B}(\mathcal{H})$ with property $\left(\mathbb{A}_{1}(r)\right)$ for some $r>0$. Then every WOT-closed subspace of $\mathcal{A}$ is hyper-reflexive.

Corollary 2.3. Every WOT-closed subspace of $H^{\infty}(\mathbb{T})$, as an operator algebra on $H^{2}(\mathbb{T})$, is hyper-reflexive.

We remark that the proof of Theorem 2.1 is rather subtle, whereas the hyperreflexivity of $H^{\infty}(\mathbb{T})$ as an operator algebra on $L^{2}(\mathbb{T})$ is a consequence of the hyperreflexivity of $L^{\infty}(\mathbb{T})$ on $L^{2}(\mathbb{T})$ and the elementary Theorem 2.2.

The following theorem is due to Bercovici and applies immediately to many free semigroupoid algebras.

Theorem 2.4 (Bercovici [3]). Suppose that $\mathcal{A}$ is a WOT-closed subspace of $\mathcal{B}(\mathcal{H})$ such that there exist two isometries in the commutant of $\mathcal{A}$ with orthogonal ranges. Then $\mathcal{A}$ is hyper-reflexive, with constant at most 3.

The next theorems are immediate consequences of this double isometry theorem and the fact that the commutant of a free semigroup algebra, or free semigroupoid algebra, is the companion algebra determined by the partial isometries $R_{e}$ for which $R_{e} \xi_{u}=\xi_{u e}$.

Theorem 2.5 (Davidson and Pitts [8]). The free semigroup algebras $\mathcal{L}_{n}$, for $2 \leq$ $n \leq \infty$, are hyper-reflexive.

Theorem 2.6 (Kribs and Power [10]). Let $G$ be a directed graph such that from every vertex $v$ there are at least two distinct directed cycles based at $v$. Then the free semigroupoid algebra $\mathcal{L}_{G}$ is hyper-reflexive.

Remark. Bercovici obtained hyper-reflexivity for a larger class of spaces than those given in Theorem 2.4, namely those with property $X_{\theta, \gamma}$. This property is defined as follows. For $x, y \in \mathcal{H}$ let $[x \otimes y]_{\mathcal{M}}$ denote the restriction of the elementary functional to the space $\mathcal{M}$. Given a number $\theta>0$ the set $\mathcal{X}_{\theta}(\mathcal{M})$ consists of all norm continuous functionals $\phi$ on $\mathcal{M}$ with the following property: for every finite set $\mathcal{F} \subset \mathcal{H}$ and every positive number $\epsilon$, there exist vectors $x, y \in \mathcal{H}$ such that:

(1) $\|x\|,\|y\| \leq 1$

(2) $\left\|\phi-[x \otimes y]_{\mathcal{M}}\right\| \leq \theta+\epsilon$; 
(3) $\left\|[x \otimes f]_{\mathcal{M}}\right\|+\left\|[f \otimes y]_{\mathcal{M}}\right\|<\epsilon$ for $f \in \mathcal{F}$.

It is well known that $\mathcal{X}_{\theta}(\mathcal{M})$ is norm closed, convex and balanced. For $\gamma>0$, the space $\mathcal{M}$ is said to have property $X_{\theta, \gamma}$ if the set $\mathcal{X}_{\theta}(\mathcal{M})$ contains all the WOTcontinuous functionals on $\mathcal{M}$ with norm no greater than $\gamma$.

We now consider spaces of operators with various forms of block matrix structure.

Let us say that a WOT-closed space of operators $\mathcal{S}$ is a block matrix space if there exist two families of projections $\mathcal{P}=\left\{P_{i} ; i=1, \ldots, r\right\}$ and $\mathcal{Q}=\left\{Q_{i} ; i=1, \ldots, s\right\}$ which partition the identity operator, and a subset $\mathcal{F} \subset \mathcal{Q} \times \mathcal{P}$ such that

$$
\mathcal{S}=\{T \in \mathcal{B}(\mathcal{H}): Q T P=0, \quad \text { for all }(Q, P) \in \mathcal{F}\} .
$$

The following proposition is elementary.

Proposition 2.7. Let $\mathcal{S}$ be a block matrix space defined by the family $\mathcal{F}$. Then $\mathcal{S}$ is hyper-reflexive with constant not exceeding $n$ where $n=|\mathcal{F}|$.

Proposition 2.8. Suppose that $\mathcal{S}$ is a hyper-reflexive space and let $S_{0}$ be a WOTclosed subspace of $\mathcal{S}$. Then $\mathcal{S}_{0}$ is hyper-reflexive if there exists a constant $C>0$ such that:

$$
d\left(X, \mathcal{S}_{0}\right) \leq C \beta_{\mathcal{S}_{0}}(X) \quad \text { for all } X \in \mathcal{S} .
$$

Proof. For a given space $\mathcal{S}$ write $\mathcal{F}_{\mathcal{S}}=\mathcal{P} \times \mathcal{Q}$ for the set of pairs of projections annihilating $\mathcal{S}$. Since $\mathcal{S}_{0} \subset \mathcal{S}$, we have $\mathcal{F}_{\mathcal{S}} \subset \mathcal{F}_{\mathcal{S}_{0}}$ and so, for $T$ in $\mathcal{B}(\mathcal{H}), \beta_{\mathcal{S}}(T) \leq$ $\beta_{\mathcal{S}_{0}}(T)$. It follows that there exists $S \in \mathcal{S}$ such that $\|T-S\| \leq C_{1} \beta_{\mathcal{S}_{0}}(T)$ where $C_{1}$ is the hyper-reflexivity constant of $\mathcal{S}$. Thus

$$
d\left(T, \mathcal{S}_{0}\right) \leq\|T-S\|+d\left(S, \mathcal{S}_{0}\right) \leq C_{1} \beta_{\mathcal{S}_{0}}(T)+d\left(S, \mathcal{S}_{0}\right),
$$

and so $\mathcal{S}_{0}$ is hyper-reflexive with constant no greater than $C_{1}+C$.

We now give an application of these elementary propositions to subspaces of block matrix spaces.

Proposition 2.9. Let $\mathcal{M}$ be a WOT-closed subspace of $\mathcal{B}(\mathcal{H})$ which is contained in a block matrix space associated with the family $\mathcal{F} \subset \mathcal{Q} \times \mathcal{P}$. Moreover, suppose that:

(1) $\mathcal{M}=\Sigma_{(Q, P) \in \mathcal{F}} \oplus Q \mathcal{M} P$.

(2) For each $(Q, P) \in \mathcal{G}$ the subspace $Q \mathcal{M P}$ is hyper-reflexive.

Then the space $\mathcal{M}$ is hyper-reflexive.

Proof. The space $\mathcal{M}$ is a subspace of the block subspace $\mathcal{S}=\Sigma_{(Q, P) \in \mathcal{F}} \oplus Q \mathcal{B}(\mathcal{H}) P$ and so, by the preceding two propositions, it is enough to prove that $d(T, \mathcal{M}) \leq$ $C \beta_{\mathcal{M}}(T)$ for $T \in \mathcal{S}$. Since the entries in the matrix decomposition of $\mathcal{M}$ are independent and $\beta_{\mathcal{M}}(Q T P) \leq \beta_{\mathcal{M}}(T)$, it is enough to prove the inequality for $T \in Q \mathcal{B}(\mathcal{H}) P$ for any pair $(Q, P)$. But the hyper-reflexivity of $Q \mathcal{M} P$ gives a constant $C>0$ such that $d(T, Q \mathcal{M} P) \leq C \beta_{Q \mathcal{M} P}(T)$. So $d(T, \mathcal{M})=d(T, Q \mathcal{M} P) \leq$ $C \beta_{Q \mathcal{M} P}(T) \leq C \beta_{\mathcal{M}}(T)$, where the last inequality holds since the annihilating set $\mathcal{F}_{Q \mathcal{M} P}$ for $Q \mathcal{M} P$ contains $\mathcal{F}_{\mathcal{M}}$.

The following corollary now follows from Davidson's theorem for $H^{\infty}(\mathbb{T})$.

Corollary 2.10. Let $\mathcal{M} \subseteq M_{n}(\mathbb{C})$ be a bimodule for the space $D_{n}$ of diagonal matrices. Then the space $H^{\infty}(\mathbb{T}) \otimes \mathcal{M}$, as a space of operators on $H^{\infty}(\mathbb{T}) \otimes \mathbb{C}^{n}$, is hyper-reflexive. 
We now turn to matrix spaces and algebras where the matrix entries are not independent. We start with a straightforward application of Bercovici's theorem to give a short new proof of a known fact (see [5]).

Proposition 2.11. Let $\mathcal{A}$ be a WOT-closed subspace of $\mathcal{B}(\mathcal{H})$. Then the infinite ampliation $\mathcal{A}^{(\infty)}$ is a hyper-reflexive subspace of $\mathcal{B}\left(\mathcal{H}^{(\infty)}\right)$.

Proof. The space $\mathcal{A}^{(\infty)}$ is equal to $\mathcal{A} \otimes \mathbb{C} I$ acting on $\mathcal{H} \otimes \ell^{2}(\mathbb{Z})$ and so the commutant contains all operators of the form $I \otimes W$ with $W$ in $\mathcal{B}\left(\ell^{2}(\mathbb{Z})\right.$. In particular, there are two isometries in the commutant with orthogonal ranges and so Bercovici's theorem applies.

The next proposition concerns what might be termed a partial inflation of operator spaces.

Proposition 2.12. Let $\mathcal{A}$ be a hyper-reflexive subspace of $\mathcal{B}(\mathcal{H})$ and let $\mathcal{M}$ be an invariant subspace for $\mathcal{A}$. Then, for any given positive integer $n$, the space

$$
\tilde{\mathcal{A}}=\left\{A \oplus A_{\mid \mathcal{M}}^{(n)} ; A \in \mathcal{A}\right\}
$$

is hyper-reflexive.

Proof. We start with $n=1$. Since the space $\mathcal{S}=\mathcal{B}(\mathcal{H}) \oplus \mathcal{B}(\mathcal{M})$ is a hyperreflexive subspace of $\mathcal{B}(\mathcal{H} \oplus \mathcal{M})$ it follows from Proposition 2.8 that it is sufficient to show that there exists a constant $C$ such that $d(\tilde{X}, \tilde{\mathcal{A}}) \leq C \beta_{\tilde{\mathcal{A}}}(\tilde{X})$ where $\tilde{X}=$ $X \oplus Y, X \in \mathcal{B}(\mathcal{H})$ and $Y \in \mathcal{B}(\mathcal{M})$. We consider the Hilbert space decomposition $\tilde{\mathcal{H}}=\mathcal{H} \oplus \mathcal{M}=\left(\mathcal{H}_{1} \oplus \mathcal{M}\right) \oplus \mathcal{M}$ and the projection defined by the matrix

$$
P=\frac{1}{2}\left(\begin{array}{ccc}
0 & 0 & 0 \\
0 & I & I \\
0 & I & I
\end{array}\right)
$$

Relative to the decomposition $\mathcal{H}_{1} \oplus \mathcal{M}$ write

$$
X=\left(\begin{array}{cc}
X_{1} & 0 \\
X_{2} & X_{3}
\end{array}\right)
$$

so that

$$
P^{\perp} \tilde{X} P=\frac{1}{4}\left(\begin{array}{ccc}
0 & 0 & 0 \\
0 & X_{3}-Y & X_{3}-Y \\
0 & Y-X_{3} & Y-X_{3}
\end{array}\right) \text { and } \beta_{\tilde{\mathcal{A}}}(\tilde{X}) \geq \frac{1}{4}\left\|X_{3}-Y\right\|
$$

Now write

$$
\tilde{X}=\left(\begin{array}{cc}
X & 0 \\
0 & X_{3}
\end{array}\right)+\left(\begin{array}{cc}
0 & 0 \\
0 & Y-X_{3}
\end{array}\right) \text { and set } X^{\prime}=\left(\begin{array}{cc}
X & 0 \\
0 & X_{3}
\end{array}\right)
$$

Then

$$
d(\tilde{X}, \tilde{\mathcal{A}}) \leq d\left(X^{\prime}, \tilde{\mathcal{A}}\right)+4 \beta_{\tilde{\mathcal{A}}}(\tilde{X})
$$

Also $\left\|X^{\prime}-A \oplus A_{\mid \mathcal{M}}\right\|=\|X-A\|$. Since $\mathcal{A}$ is hyper-reflexive with constant $C_{\mathcal{A}}$, we have

$$
d\left(X^{\prime}, \tilde{\mathcal{A}}\right)=d(X, \mathcal{A}) \leq C_{\mathcal{A}} \beta_{\mathcal{A}}(X) \leq C_{\mathcal{A}} \beta_{\tilde{\mathcal{A}}}(\tilde{X})
$$

The last inequality holds since $\mathcal{F}_{\tilde{\mathcal{A}}} \supset \mathcal{F}_{\mathcal{A}}$ and $X$ is a summand of $\tilde{X}$. Finally, we have

as desired.

$$
d(\tilde{X}, \tilde{\mathcal{A}}) \leq\left(C_{\mathcal{A}}+4\right) \beta_{\tilde{\mathcal{A}}}(\tilde{X})
$$


Suppose now that $\tilde{\mathcal{A}}_{n}=\left\{A \oplus A_{\mid \mathcal{M}}^{(n)} ; A \in \mathcal{A}\right\}$ is hyper-reflexive. Since $\tilde{A}_{n+1}=$ $A \oplus A_{\mid \mathcal{M}}^{(n+1)}$ is unitarily equivalent to $\tilde{A}_{n} \oplus\left(\tilde{A}_{n}\right)_{\mid 0 \oplus \mathcal{M}}$, it follows that $\tilde{\mathcal{A}}_{n+1}$ is also hyper-reflexive.

The next proposition deals with infinite partial ampliations:

Proposition 2.13. Let $\mathcal{A}$ be a WOT-closed operator algebra which is hyper-reflexive and let $\mathcal{M}$ be an invariant subspace for $\mathcal{A}$. Then the operator algebra

$$
\tilde{\mathcal{A}}=\left\{A \oplus A_{\mid \mathcal{M}}^{(\infty)} ; A \in \mathcal{A}\right\}
$$

is hyper-reflexive.

Proof. Since $\mathcal{B}(\mathcal{H}) \oplus \mathcal{A}_{\mid \mathcal{M}}^{(\infty)}$ is hyper-reflexive, as a simple consequence of Proposition 2.11 , it is enough in view of Proposition 2.8 to derive the distance formula for elements of the form $X \oplus A_{\mid \mathcal{M}}^{(\infty)}, X \in \mathcal{B}(\mathcal{H}), A \in \mathcal{A}$. Set $\tilde{\mathcal{A}}_{1}=\left\{A \oplus A_{\mid \mathcal{M}} ; A \in \mathcal{A}\right\}$, which is hyper-reflexive by the previous proposition, with constant $C_{1}$. Then

$$
d\left(X \oplus A_{\mid \mathcal{M}}^{(\infty)}, \tilde{\mathcal{A}}\right)=d\left(X \oplus A_{\mid \mathcal{M}}, \tilde{\mathcal{A}}_{1}\right) \leq C_{1} \beta_{\tilde{\mathcal{A}}_{1}}\left(X \oplus A_{\mid \mathcal{M}}\right) \leq C_{1} \beta_{\tilde{\mathcal{A}}}\left(X \oplus A_{\mid \mathcal{M}}^{(\infty)}\right)
$$

which completes the proof.

\section{HyPER-REFLEXIVITY OF FREE SEMIGROUPOID ALGEBRAS}

We now consider a finite directed graph $G$ with semigroupoid algebra $\mathcal{L}_{G}$ acting on the Fock space $\mathcal{H}_{G}$ and, as we have intimated in the introduction, we shall show that $\mathcal{L}_{G} \otimes \mathcal{B}(\mathcal{K})$ is hyper-reflexive, for $\mathcal{K}$ a separable Hilbert space.

It does not seem to be known whether the hyper-reflexivity of an operator algebra $\mathcal{A}$ entails that of $\mathcal{A} \otimes \mathcal{B}(\mathcal{K})$, even allowing for a different hyper-reflexivity constant. We find it convenient in the subsequent discussion to say that $\mathcal{A}$ is completely hyperreflexive if this apparently stronger assertion holds. That completely hyper-reflexive spaces are hyper-reflexive is elementary.

Our proof uses an induction argument with an induction step that describes a block matrix structure of $\mathcal{L}_{G}$ in terms of $\mathcal{L}_{G^{\prime}}$ where $G^{\prime}$ is a subgraph of $G$ obtained by deleting a single edge $e$. We can in fact restrict attention to the case where $e$ does not lie on any directed loop path with source vertex equal to the source of $e$. In the block matrix decomposition there is column subspace of the form $\mathcal{S} \otimes \ell^{2}$ with $\mathcal{S} \subseteq \mathcal{L}_{G^{\prime}}$ a hyper-reflexive space, and so we need complete hyper-reflexivity for $\mathcal{L}_{G^{\prime}}$ even to deduce that $\mathcal{L}_{G}$ is hyper-reflexive.

We shall make use of the following strengthened form of Davidson's theorem for $H^{\infty}(\mathbb{T})$ on $H^{2}(\mathbb{T})$ the proof of which is an entirely routine adaptation of the proof given in $[6]$.

Theorem 3.1. The algebra $H^{\infty}(\mathbb{T})$ realized as the algebra of Toeplitz operators acting on $H^{2}(\mathbb{T})$ is completely hyper-reflexive, with constant at most 19.

We first isolate the case when $G$ is a cycle; in this case we write $G=C_{n}$, where $n$ is the number of vertices in $G$. The corresponding Fock space will be denoted by $\mathcal{H}_{n}$.

Theorem 3.2. For any integer $n>0$, the cycle algebra $\mathcal{L}_{C_{n}}$ is completely hyperreflexive. 
Proof. If $n=1$, then $\mathcal{L}_{C_{1}}=H^{\infty}(\mathbb{T})$, acting on $H^{2}$, which is completely hyperreflexive. For $n \geq 2$, the Fock space $\mathcal{H}_{n}$ decomposes as an orthogonal sum of the ranges of the projections $L_{v}$ for $v \in \mathcal{V}\left(C_{n}\right)$ :

$$
\mathcal{H}_{n}=\bigoplus_{i=1}^{n} L_{v_{i}} \mathcal{H}
$$

Identify each space $L_{v_{i}} \mathcal{H}$ with the Hardy space $H^{2}(\mathbb{T})$. Then it is straightforward to verify that $\mathcal{L}_{C_{n}}$ is unitarily equivalent to the matrix function algebra acting on $H^{2} \oplus \cdots \oplus H^{2}$, with elements of the form

$$
\left(\begin{array}{ccccc}
f_{11}\left(z^{n}\right) & z^{n-1} f_{12}\left(z^{n}\right) & z^{n-2} f_{13}\left(z^{n}\right) & \cdots & z f_{1 n}\left(z^{n}\right) \\
z f_{21}\left(z^{n}\right) & f_{22}\left(z^{n}\right) & z^{n-1} f_{23}\left(z^{n}\right) & \cdots & z^{2} f_{2 n}\left(z^{n}\right) \\
z^{2} f_{31}\left(z^{n}\right) & z f_{32}\left(z^{n}\right) & f_{33}\left(z^{n}\right) & \cdots & \vdots \\
\vdots & \vdots & \vdots & \ddots & \vdots \\
z^{n-1} f_{n 1}\left(z^{n}\right) & z^{n-2} f_{n 2}\left(z^{n}\right) & \cdots & \cdots & f_{n n}\left(z^{n}\right)
\end{array}\right),
$$

where each $f_{i j} \in H^{\infty}(\mathbb{T})$. Thus the algebra $\mathcal{L}_{C_{n}}$ is identifiable with a block operator matrix algebra with copies of $H^{\infty}\left(z^{n}\right)$ on the diagonal and with off-diagonal spaces of the form $z^{j} H^{\infty}\left(z^{n}\right)$, where $H^{\infty}\left(z^{n}\right)$ denotes the subalgebra spanned by the powers $z^{n k}, k=0,1,2, \ldots$.

The space $H^{\infty}\left(z^{n}\right)$ is unitarily equivalent to the ampliation algebra $H^{\infty}(\mathbb{T})^{(n)}=$ $H^{\infty}(\mathbb{T}) \otimes \mathbb{C} I$ acting on $H^{2}(\mathbb{T}) \otimes \mathbb{C}^{n}$. Also, it is elementary to see that finite ampliations of a hyper-reflexive algebra are hyper-reflexive. Thus $H^{\infty}\left(z^{n}\right)$ on $H^{2}(\mathbb{T})$ is completely hyper-reflexive. Also, it is elementary that if $\mathcal{S}$ is a completely hyperreflexive space and $U$ is an isometry (such as $T_{z^{j}} \otimes I$ in the present context), then $U \mathcal{S}$ is a completely hyper-reflexive space. Thus $z^{j} H^{\infty}\left(z^{n}\right)$ on $H^{2}(\mathbb{T})$ is completely hyper-reflexive for each $j=1, \ldots, n$. It follows now, from Proposition 2.9, for examples, that the matrix function operator algebra above is completely hyper-reflexive, as required.

We now turn to the following key induction step.

Proposition 3.3. Let $G$ be a graph with directed edge $e=y e x$, for which there is no directed path from vertex $y$ to vertex $x$ and let $G^{\prime}$ be the graph where e has been deleted : $G^{\prime}=G \backslash$ e. If $\mathcal{L}_{G^{\prime}}$ is completely hyper-reflexive, then so too is $\mathcal{L}_{G}$.

Proof. We write $\mathcal{H}=\mathcal{H}_{G}$ and $\mathcal{H}^{\prime}=\mathcal{H}_{G^{\prime}}$ for the Fock spaces associated with $G$ and $G^{\prime}$ respectively. Let $\mathcal{H}^{\prime}$ be viewed as the natural subspace of $\mathcal{H}$, that is, the subspace spanned by the basis elements $\xi_{w}$ for which $e$ does not appear in $w$. We write $e \notin w$ to indicate this. With respect to the decomposition $\mathcal{H}=\mathcal{H}^{\prime} \oplus \mathcal{K}$ note that $\mathcal{K} \subseteq \operatorname{ker} L_{e}$ and that the operator matrix for $L_{e}$ is strictly lower triangular. Also, for any other edge $g \in \mathcal{E}(G), L_{g}$ has block diagonal matrix. It follows that for a given word $w \in \mathbb{F}_{+}(G), L_{w}$ is block diagonal if $e \notin w$, and is lower triangular if $e \in w$. Thus $\mathcal{L}_{G}$ is the direct sum of its diagonal subalgebra, which we denote by $\mathcal{D}$, and its lower triangular subalgebra, $\mathcal{T}$ say.

We now show that both $\mathcal{D}$ and $\mathcal{T}$ are completely hyper-reflexive and it follows readily (by a simple adaptation of the proof of Proposition 2.9 for example) that $\mathcal{D}+\mathcal{T}$ is completely hyper-reflexive. 
Consider first the decomposition of $\mathcal{K}$ associated with the different words in $G^{\prime}$ ending at $x$ :

$$
\mathcal{K}=\bigoplus_{i=1}^{n} \mathcal{K}^{i}, \quad \mathcal{K}^{i} \simeq \mathcal{H}_{y}
$$

where $\mathcal{H}_{y}=\operatorname{span}\left\{\xi_{w} ; \quad w=w y\right\}$ and $n$ is the cardinality of $\left\{w \in \mathcal{W}\left(G^{\prime}\right) ; \quad w=\right.$ $x w\}$. (Possibly $n=\infty$.) The block diagonal algebra $\mathcal{D}$ is therefore unitarily equivalent to the algebra

$$
\left\{A \oplus \bigoplus_{i=1}^{n} A_{\mid \mathcal{K}^{i}} \quad A \in \mathcal{L}_{G^{\prime}}\right\} .
$$

In view of the complete hyper-reflexivity of $\mathcal{L}_{G^{\prime}}$ it follows from Propositions 2.12 and 2.13 that this algebra is completely hyper-reflexive.

To see the complete hyper-reflexivity of the lower triangular subalgebra $\mathcal{T}$, we use a different orthogonal decomposition of $\mathcal{K}$ namely the one obtained from the wandering subspace

$$
\mathcal{K}_{0}=L_{e} \mathcal{H}\left(=L_{e} L_{e}^{*} \mathcal{H} \simeq L_{x} \mathcal{H}=L_{x} \mathcal{H}^{\prime}\right)
$$

that is,

$$
\mathcal{K}=\bigoplus_{w=w y} L_{w} \mathcal{K}_{0} .
$$

With respect to the associated block matrix structure and the natural identification of each summand $L_{w} \mathcal{K}_{0}$ with $\mathcal{K}_{0}$ the restriction operator $L_{w \mid \mathcal{K}}$ is a block matrix whose entries are either the identity or zero. Now, with respect to the decomposition

$$
\mathcal{H}=\left(\mathcal{H}^{\prime} \ominus L_{x} \mathcal{H}^{\prime}\right) \oplus L_{x} \mathcal{H}^{\prime} \oplus \mathcal{K}_{0} \oplus\left(\mathcal{K} \ominus \mathcal{K}_{0}\right),
$$

the operator $L_{e}$ has the operator matrix

$$
L_{e}=\left(\begin{array}{cccc}
0 & 0 & 0 & 0 \\
0 & 0 & 0 & 0 \\
0 & I & 0 & 0 \\
0 & 0 & 0 & 0
\end{array}\right) .
$$

Let us write the restricted algebra $\mathcal{D}_{\mid \mathcal{H}^{\prime}}$ which is isomorphic to $\mathcal{L}_{G^{\prime}}$ in terms of its $2 \times 2$ block structure as

$$
\mathcal{D}_{\mid \mathcal{H}^{\prime}}=\left(\begin{array}{cc}
\mathcal{A}_{1} & \mathcal{A}_{2} \\
\mathcal{B} & \mathcal{C}
\end{array}\right)
$$

Since $\mathcal{L}_{G^{\prime}}$ is completely hyper-reflexive, by assumption, it follows that the space of matrices

$$
L_{x} \mathcal{D}=\left(\begin{array}{ll}
0 & 0 \\
\mathcal{B} & \mathcal{C}
\end{array}\right)
$$

is completely hyper-reflexive. Also, $\mathcal{T}$ can be identified with the lower triangular space

$$
\mathcal{T} \simeq\left(\begin{array}{cc}
0 & 0 \\
{[\mathcal{B} \mathcal{C}] \otimes l_{+}^{2}(E)} & 0
\end{array}\right)
$$

where $E=\mathcal{W}(G) y$ is the set of words $w$ in $G$ of the form $w=w y$, and so it follows that $\mathcal{T}$ is completely hyper-reflexive, as required.

Theorem 3.4. For a finite directed graph $G$ the free semigroupoid algebra $\mathcal{L}_{G}$ is completely hyper-reflexive. 
Proof. If $G$ is the single cycle graph $C_{n}$, then $\mathcal{L}_{G}$ is completely hyper-reflexive as we saw above, so we may assume that $G$ is not of this form.

Suppose first that $G$ is a transitive directed graph, that is, that every pair of vertices lies on a directed cycle. The commutant of $\mathcal{L}_{G} \otimes \mathcal{B}(\mathcal{K})$ contains the ampliated commutant $\left(\mathcal{L}_{G}\right)^{\prime} \otimes \mathbb{C} I$. Also, $\left(\mathcal{L}_{G}\right)^{\prime}$ is isomorphic to $\mathcal{L}_{G^{t}}$ where the transpose graph $G^{t}$ is also transitive. (See [10.) In view of our initial assumption every vertex of the transitive graph, and its transpose, lies on two distinct cycles. An elementary argument, given in [10, shows that in this case there is a pair of isometries in $\mathcal{L}_{G^{t}}$ with orthogonal ranges. Thus there is a pair of isometries in the commutant of $\mathcal{L}_{G} \otimes \mathcal{B}(\mathcal{K})$ with orthogonal ranges and Bercovici's theorem shows that $\mathcal{L}_{G}$ is completely hyper-reflexive.

Suppose now, by way of induction, that $\mathcal{L}_{G}$ is completely hyper-reflexive for all graphs with $n$ or fewer edges, and let $G$ have $n+1$ edges. If $G$ is transitive, then it is completely hyper-reflexive, by the above, and so we may assume that there is an edge $e=(x, y)$ for which there is no path from $x$ to $y$. The induction hypothesis and Proposition 3.3 complete the proof.

\section{REFERENCES}

[1] A. Arias, G. Popescu, Factorization and reflexivity on Fock spaces, Integral Equations Operator Theory 23 1995, 268-286. MR1356335 (97e:47066)

[2] W.B. Arveson, Ten lectures on operator algebras, CBMS Regional Conference Series in Mathematics, 55, American Mathematical Society, 1984. MR0762819 (86d:47055)

[3] H. Bercovici, Hyper-reflexivity and the factoriation of linear functionals, J. Func. Anal., 158:242-252, 1998. MR1641578 (99g:47105)

[4] C.M. Pearcy, H. Bercovici and C. Foias, Dual algebras with application to invariant subspaces and dilation theory, volume 56, CBMS Regional Conf. Ser. in Math., Amer. Math. Soc., Providence, 1985. MR0787041 (87g:47091)

[5] K.R. Davidson, Nest algebras, Triangular forms for operator algebras on Hilbert space, Pitman Research Notes in Mathematics Series, 191, Longman Scientific and Technical, Harlow; copublished in the United States with John Wiley and Sons, Inc., New York, 1988. MR.0972978 (90f:47062)

[6] K.R. Davidson. The distance to the analytic Toeplitz operators, Illinois J. Math., 31:265-273, 1987. MR0882114 (88e:47050)

[7] K.R. Davidson, E. Katsoulis and D.R. Pitts, The structure of free semigroup algebras, J. reine angew. Math. 533 (2001), 99-125. MR1823866 (2002a:47107)

[8] K.R. Davidson and D.R. Pitts, Invariant subspaces and hyper-reflexivity for free semi-group algebras, Proc. London Math. Soc. 78 (1999), 401-430. MR 1665248 (2000k:47005)

[9] K.R. Davidson and D.R. Pitts, The algebraic structure of non-commutative analytic Toeplitz algebras, Math. Ann. 311 (1998), 275-303. MR.1625750 (2001c:47082)

[10] D.W. Kribs and S.C. Power, Free semigroupoid algebras, J. Ramanujan Math. Soc., 19 (2004), 117-159. MR2076898(2005c:47106)

[11] D.W. Kribs and S.C. Power, Partly Free Algebras From Directed Graphs, Operator Theory: Advances and Applications, 149 (2004), 381-393. MR.2063759 (2005c:47105)

[12] G. Popescu, Noncommuting disc algebras and their representations, Proc. Amer. Math. Soc. 124 (1996), 2137-2148. MR.1343719 (96k:47077)

[13] G. Popescu, A generalization of Beurling's theorem and a class of reflexive algebras, J. Operator Theory 41 (1999), 391-420. MR1681580 (2000h:47108)

University of Bordeaux I, LaBAG 351, cours de la Liberation, F-33405 Talence, Cedex, France

E-mail address: jaeck@math.u-bordeaux1.fr

Department of Mathematics and Statistics, Lancaster University, Lancaster, LanCASHIRE LA1, ENGLAND

E-mail address: s.power@lancaster.ac.uk 